\title{
Electron Microscopic Studies of the Eukaryotic pre-Replicative Mcm2-7 complex
}

\author{
Fabienne Beuron ${ }^{1}$, Max Douglas ${ }^{2}$, Jordi Fridgola ${ }^{2}$, John Diffley ${ }^{2}$ and Edward Morris ${ }^{1}$ \\ 1. The Institute of Cancer Research, Division of Structural Biology, London UK. \\ 2. Cancer Research UK London Research Institute, Chromosome Replication Laboratory, South Mimms \\ UK.
}

The minichromose maintenance (Mcm2-7) proteins are key subunits of a large molecular complex that assembles at the origins of replication before the onset of DNA replication in eukaryotic cells. They are members of the AAA+ superfamily and are thought to constitute the replicative helicase. We have reconstituted the Mcm2-7 loading reaction in vitro from purified proteins (Orc1-6, Cdt1, Cdc6, Mcm27) and have characterized the structure of loaded MCM2-7 complexes using single particle electron microscopy [1].

The origin recognition complex (ORC) is first recruited to the replication origin. ORC recruits Cdc6 and Cdt1. ORC, Cdc6 and Cdt1 act together to load multiple minichromosome maintenance Mcm2-7 protein hexamers onto the origin, which licenses the DNA for replication. In our reconstitution assay [2], DNA-beads incubated with purified proteins in the presence of ATP, bind ORC and Mcm2-7 whilst Cdc6 and Cdt1 are released. When a high salt wash is applied to this reaction, ORC is removed from the DNA but Mcm2-7 remains bound. In the course of the loading reaction Mcm2-7 complexes are formed into stable DNA-bound double-hexameric Mcm2-7 complexes that exhibit a four-tiered ring structure in a head-to-head orientation. We have expanded on our initial study [1] and in the view to achieve high resolution structural information using cryo-electron microscopy we have first focused on obtaining a good starting model using negative stain data obtained at $200 \mathrm{kV}$ with a FEI TF20 microscope. A typical field of a micrograph (figure 1A) shows the high degree of homogeneity of the sample.

Our data set is constituted exclusively of side views with good signal to noise at the raw particle level (figure 1B). For the purpose of generating the reference model classes obtained by iterative stable alignment and clustering (ISAC) [3] were calculated (figure 2A). The quality of the data set is reflected in the good level of details in the resulting class averages as exemplified for instance by the four discreet domains observed at the interface between the two hexamers.

The map presented here (figure 2B) was obtained by assigning Euler angles to a data set of 2,807 side views by projection matching using our new starting reference. During processing only in plane 2 fold rotation was applied and no assumption about the rotational symmetry around the long axis was. The double hexamer is a complex barrel structure of $15 \times 23 \mathrm{~nm}$. Examination of the longitudinal sections through the $3 \mathrm{D}$ reconstruction reveal that the central channel is filled with densities compatible in size with double stranded DNA (figure 2C). Furthermore there is no continuity between the cavities of the 2 hexamers. Although no assumption about the rotational symmetry around the long axis was made during the analysis the 3D map obtained presents a hexameric outline at the level of the N-terminal tier ring (not shown).

This structure accommodates very well 12 copies of the near full length crystal structure of the Sso MCM [4] (figure 2C). The $\mathrm{N}$ terminal tier ring presents additional outward extensions whereas the $\mathrm{C}$ terminal outer ring has 2 protrusions pointing upward close to the central channel. These lateral 
protuberances can accommodate the extensive $\mathrm{N}$ terminal tails of the $\mathrm{Mcm} 2, \mathrm{Mcm} 4$ and $\mathrm{Mcm} 6$. Similarly the distal tips could contain the $\mathrm{C}$ terminal tails of $\mathrm{Mcm} 3$ and $\mathrm{Mcm} 6$. Interestingly the two hexamers are not in direct register. Connecting densities are present at different radial position between the $\mathrm{N}$ termini at the interface between the two hetero-hexamers. We will seek to determine the register between the two hetero-hexamers using N-terminal tagging of Mcm3 with MBP.

These findings will help shed light on the mechanism of replicative helicase loading at eukaryotic origins and may suggest a mechanism for the establishment of bidirectional replication forks on an asymmetric origin.

[1] D Remus, F Beuron, G Tolun, JD Griffith, EP Morris, and JF Diffley, Cell 139 (2009), p. 719

[2] A Mehanna and J Diffley, Methods 57 (2012), p. 222

[3] Z Yang, Z Yang, J Fang, J Chittuluru, FJ Asturias, and PA Penczek, Structure 20 (2012), p. 237

[4] W Liu, B Pucci, M Rossi, FM Pisani, and R Ladenstein, Nucleic Acids Res. 36 (2008), p. 3235

Figure 1. A, Typical micrograph of the negatively stained preparation showing the high level of homogeneity of the sample. Example side views are indicated by arrows; scale bar represents $20 \mathrm{~nm}$. B, Gallery of manually selected double hexamers side views constituting the raw data set; scale bar represents $10 \mathrm{~nm}$.


Figure 2. A, Examples of refined classes obtained using ISAC; scale bar represents $10 \mathrm{~nm}$. B, Surface representation of one two-fold view of the double hexamer. C, In the cut open view one double stranded DNA fragment (orange) is fitted in the internal densities not accounted for by Sso Mcm crystal structure.
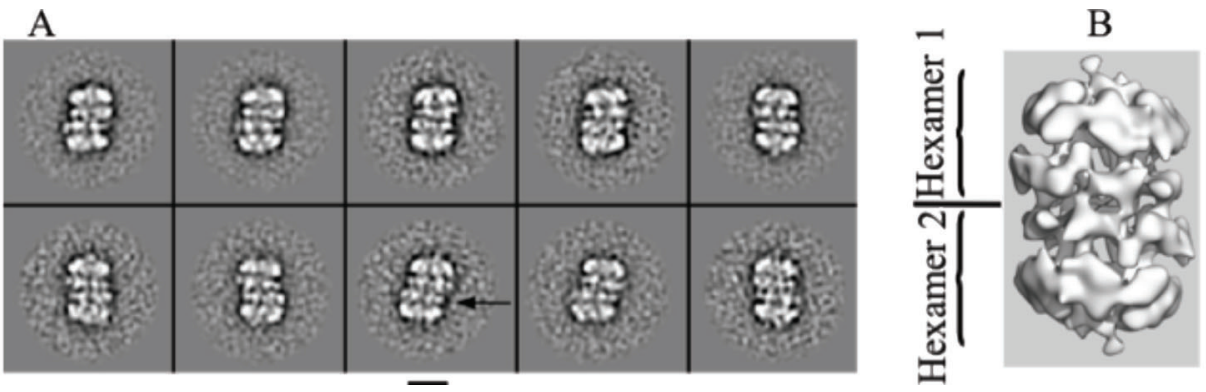

$\underline{10 \mathrm{~nm}}$

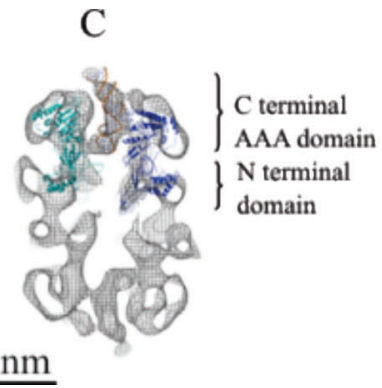

